\title{
TUNABLE-FOCUS MICROLENSES ACTUATED BY INFRARED-LIGHT-RESPONSIVE HYDROGELS WITH ENTRAPPED GOLD NANOPARTICLES FOR FIBER ENDOSCOPY
}

\author{
$X$. Zeng and H. Jiang
}

Department of Electrical \& Computer Engineering, University of Wisconsin - Madison, Madison, WI, USA

\begin{abstract}
We report on liquid microlenses with tunable focal length actuated by infrared- (IR-) light-responsive hydrogel with entrapped gold nanoparticles. A curved water-oil interface forms the liquid microlens; this water-oil meniscus is pinned at a hydrophobic-hydrophilic boundary at the top edge of an aperture. IR-light responsive hydrogel microstructures are photopatterned under ultraviolet light. The volumetric change in the hydrogel, when controlled by IR-light, regulates the pressure difference across the water-oil interface, varying its curvature and the resulting focal length of the microlens. The focal length of the microlens varies from $-17.4 \mathrm{~mm}$ (slightly divergent) to $-\infty$ (flat) and from $+\infty$ to 8.8 $\mathrm{mm}$ (convergent) in 4 seconds under IR-light irradiation. The spherical aberration of the microlens is $-0.245 \mu \mathrm{m}$.
\end{abstract}

\section{INTRODUCTION}

Fiber endoscopes [1] are important diagnostic and surgical tools. Current fiber endoscopes use non-tunable lenses at the distal tip for imaging; hence their operation requires constant skillful manual maneuver. Tunable-focus microlenses integrated at the end of endoscopes to scan the areas of interest with minimum movements of the scopes themselves would therefore greatly advance endoscopy.

However, current tunable microlens technologies [2] are not best suited for this purpose due to their relative large size, complicated fabrication and demanding actuation. Here, we demonstrate a relatively simple procedure to fabricate tunable microlenses. The microlenses are actuated by compact infrared(IR-) light-responsive hydrogel microstructures with entrapped gold nanoparticles. Patterning and actuation of the hydrogel actuators (thus focal length tuning) are both realized with light, as shown in Fig. 1; hence the microlenses are intrinsically integratable with fibers optics for fiber endoscopy.

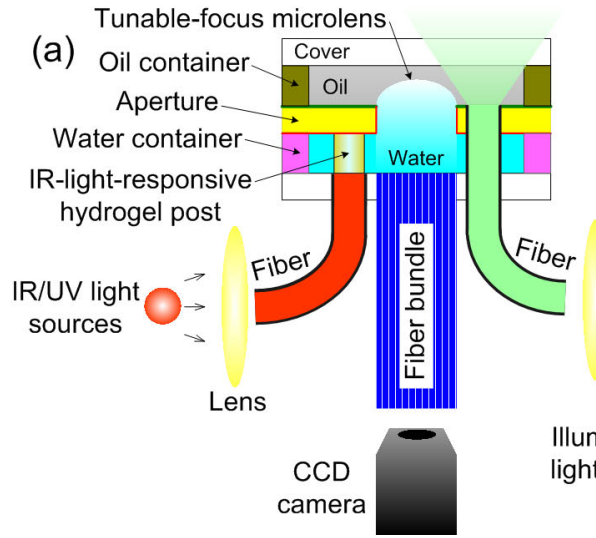

(b)
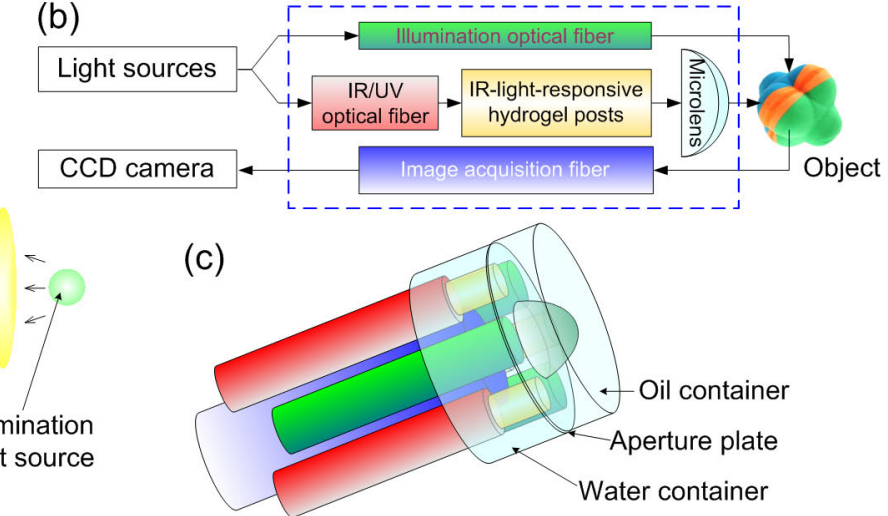

Figure 1: Schematics of potential application of IR-light-responsive tunable liquid microlenses in fiber endoscopes: (a) cross-section, (b) mechanism and (c) 3-D schematic of the distal end. The hydrogel actuators controlled by IR light tune the focal length of the liquid microlens formed by a water-oil meniscus. The hydrogel is first patterned under ultraviolet (UV) exposure, and is later controlled by IR-light, all from the same set of fibers. Another set of fibers illuminates the area of interest. Central fiber bundle guides the acquired image to image acquisition devices such as a CCD camera.

\section{PRINCIPLE AND STRUCTURES}

Fig. 2 describes the structure and mechanism of a tunable-focus microlens. An aperture plate and water container is fabricated through liquid phase photopolymerization $\left(\mathrm{LP}^{3}\right)$. A microlens is formed through a curved interface between water and oil. The sidewalls and bottom surfaces of the aperture are chemically treated hydrophilic, as shown in the red lines in Fig. 1(a), 2(c) and 2(d), while the top surfaces are naturally hydrophobic. Thus a water-oil meniscus is pinned at this hydrophobic-hydrophilic $(\mathrm{H}-\mathrm{H})$ boundary at the top edge of the aperture. Oil prevents the evaporation of water and serves as lens material along with water since the refractive index of oil (1.48) is larger than that of water (1.33).

Multiple IR-light-responsive hydrogel micro posts are formed in the water container to actuate the microlens under IR-light irradiation. IR-light-responsive hydrogel consists of a thermo-responsive reversible $N$-isopropylacrylamide (NIPAAm) hydrogel $[3,4]$ and water-soluble gold nanoparticles with distinct and strong optical absorption of IR-light, which has high heat efficiency [5]. With the IR-light turned on, the gold nanoparticles absorb the IR-light, generating heat to cause the hydrogel to contract. With the IR-light turned off, the heat dissipates and the hydrogel expands back to the original volume. The resultant net change between the hydrogel and water regulates the pressure difference across the water-oil interface. The curvature of the interface varies from divergent to convergent and the focal length of the microlens changes from negative to positive, as shown in Fig 2(c) and 2(d).

\section{FABRICATION METHODS} Synthesis of Gold Nanoparticles

The water-soluble gold nanoparticles with thiolated ligands are synthesized using Brust method [6, 7], as shown in Fig. 3(a). First, $2.19 \mathrm{~g}$ of tetraoctylammonium bromide $\left(\left[\mathrm{CH}_{3}\left(\mathrm{CH}_{2}\right)_{7}\right]_{4} \mathrm{NBr}\right)$ is dissolved in $80 \mathrm{~mL}$ of toluene, and $30 \mathrm{~mL}$ of a $30 \mathrm{mM}$ aqueous hydrogen tetrachloroaurate $\left(\mathrm{HAuCl}_{4}\right)$ solution is added. Under stirring, $100 \mathrm{mg}$ of hexanethiol is added, followed by slowly adding 
$25 \mathrm{~mL}$ of $0.4 \mathrm{M}$ aqueous sodium borohydride $\left(\mathrm{NaBH}_{4}\right)$ solution. After the mixture is stirred for 1 hour, the solvent is evaporated and the organic layer is isolated. The particles are washed three times with ethanol. The gold particles are then collected and dispersed in $10 \mathrm{~mL}$ of dichloromethane (DCM). Next, $10 \mathrm{~mL}$ of $1.82-\mathrm{mM}$ aqueous polyethylene glycol (PEG) solution is added for the exchange reaction. After 24 hours, DCM is removed under reduced pressure with mild heat and the particles with excessive PEG are redispersed in DCM and stirred for another 48 hours. The particles are again evaporated to dry, redispersed in deionized (DI) water, and centrifuged to remove insoluble particles. The concentration of gold nanoparticles in the solution is around $4.7 \mathrm{mg} / \mathrm{mL}$.

Fig. 3(b) shows the scanning electron microscope (SEM) image of the synthesized gold nanoparticles coated with PEG-thiol polymer. The diameter of the gold nanoparticles is between $10 \mathrm{~nm}$ to $20 \mathrm{~nm}$.
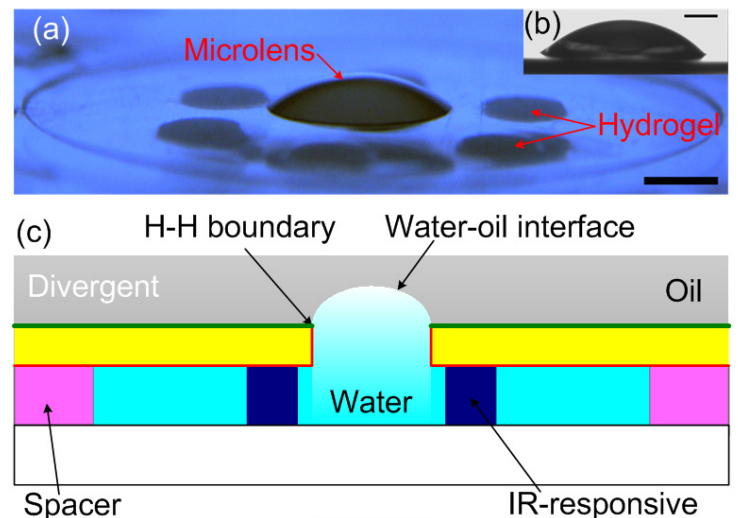

(d)

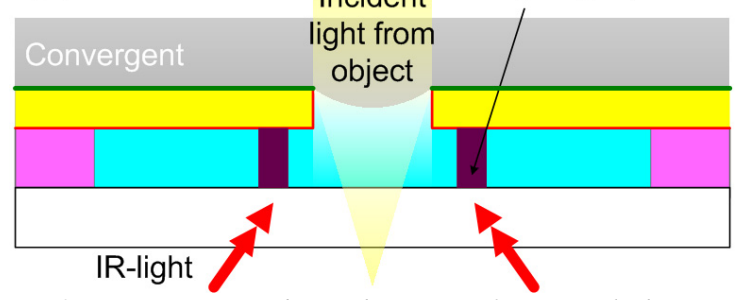

Figure 2: Structure and mechanism of an IR-light actuated tunable-focus microlens. (a) Picture of the microlens taken with a stereoscope from an oblique angle. The scale bar is $1 \mathrm{~mm}$. (b) Side profile of the water meniscus of the microlens at the starting point taken with a goniometer. The scale bar is $0.5 \mathrm{~mm}$. Oil is not yet added for a clearer image. (c) Divergent microlens with expanded hydrogel posts and (d) convergent microlens with contracted hydrogel posts under IR-light irradiation.

\section{Polymer Precursors}

Two pre-polymer solutions are used in the fabrication process: isobornyl acrylate (IBA) and IR-light-responsive hydrogel [8]. IBA pre-polymer solution is used for the structural polymers, such as aperture plate and water container. Under UV radiance, IBA pre-polymer solution is polymerized, called poly-IBA.

IR-light-responsive hydrogel pre-polymer solution consists of five components: NIPAAm $0.545 \mathrm{~g}$ as the monomer, $0.0385 \mathrm{~g}$ 2,2-dimethoxy-2-phenylace-tophenone (DMPA) as the co-monomer, $0.031 \mathrm{~g} N, N^{\prime}$-methylenebisacrylamide (NMBA) as the cross-linker, $0.75 \mathrm{~mL}$ dimethyl sulfoxide (DMSO) and $0.25 \mathrm{~mL}$ gold nanoparticles solution as the solvents. Exposure to a UV-light source causes the pre-polymer solution to harden because of the photopolymerization.

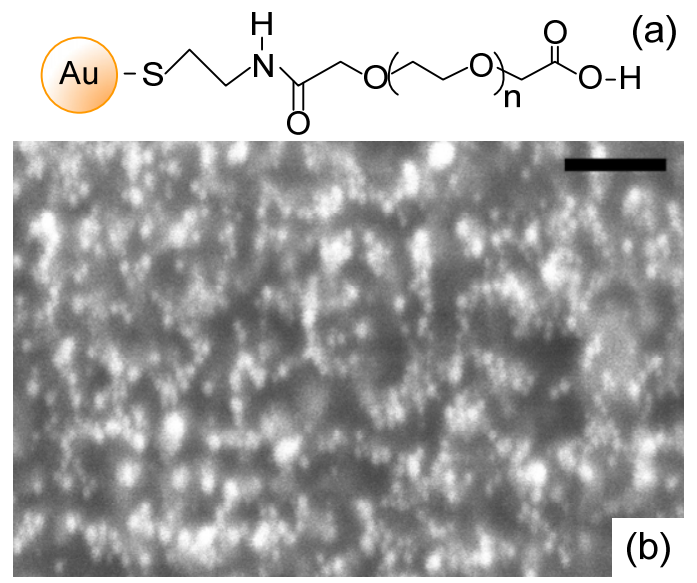

Figure 3: (a) Chemical structure of the water-soluble gold nanoparticles. (b) SEM image of the gold nanoparticles coated with PEG-thiol polymer. The scale bar represents $100 \mathrm{~nm}$.

\section{Fabrication}

Fig. 4 shows the whole process based on $\mathrm{LP}^{3}$ to fabricate the microlens [8, 9]. First, a polycarbonate cartridge cavity is filled with IBA pre-polymer solution. The cartridge cavity consists of top cartridge plates and bottom liner plates. The thickness of the cavity is defined by the spacer and is $250 \mu \mathrm{m}$, as shown in Fig. 4(a). The film photomask (Photomask I) is aligned on top of the cartridge to pattern the poly-IBA aperture in the cavity using $\mathrm{LP}^{3}$ under UV radiance (intensity, $I_{U V}=8.2 \mathrm{~mW} / \mathrm{cm}^{2}$; time, $t=23 \mathrm{~s}$ ), as shown in Fig. 4(b). The bottom liner plate is peeled off and the cartridge plate is flipped over. Next, another film mask (Photomask II) is used as the top plate and a cavity around $800 \mu \mathrm{m}$ thick is formed. A microchannel is formed on top of the aperture plate using the similar $\mathrm{LP}^{3}$ procedure $\left(I_{U V}=8.2 \mathrm{~mW} / \mathrm{cm}^{2} ; t=35 \mathrm{~s}\right)$, as shown in Fig. 4(c). Side walls and top surfaces of the poly-IBA plate are rendered with oxygen plasma (power supply $50 \mathrm{~W}$; oxygen flow rate $20.0 \mathrm{sccm}$; gas pressure 20 mtorr; treatment time $30 \mathrm{~s}$ ) from hydrophobic to hydrophilic, shown as red lines in Fig. 4(d), to form the H-H pinning boundaries. Then, the mask plate is peeled off and the poly-IBA plate is flipped over and bonded onto a glass slide, forming the water container, as shown in Fig. 4(e). Multiple IR-light-responsive hydrogel posts are fabricated in the water container with a photomask (intensity, $I_{U V}=12.5 \mathrm{~mW} / \mathrm{cm}^{2}$; time, $t=19 \mathrm{~s}$ ), as shown in Fig. 4(f). To achieve better hydrophobicity on the top surface of the aperture plate, an octadecyltrichlorosilane (OTS) solution diluted by hexadecane $(0.2 \% \mathrm{v} / \mathrm{v})$ is brushed onto it, as shown in Fig. 4(g). Finally, a polydimethylsiloxane (PDMS) ring, whose surface is treated under high frequency electric field to improve adhesion, is bonded on top of the aperture plate and covered by a glass slide to form an oil container, as shown in Fig. 4(h).

\section{EXPERIMENTS AND RESULTS \\ Spectra of Gold Nanoparticles Solution}

The absorption spectrum is measured by Edmund Industrial Optics CCD Spectrometer. The scanning wavelength range is from 400 to $1100 \mathrm{~nm}$. All liquid samples are measured in cuvets with 1 $\mathrm{mm}$ long light-path. The hydrogel with entrapped gold nanoparticles is polymerized in a cuvet under UV radiance for 10 minutes. Fig. 5 shows the absorption spectra of the gold nanoparticle solution, the polymerized IR-light-responsive hydrogel with entrapped gold nanoparticles, DI water and the IR-light source. Gold nanoparticles in the solution and entrapped in the hydrogel have high absorption of light in the IR range. 
(a)

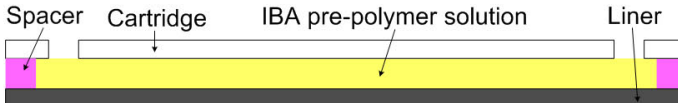

(b)

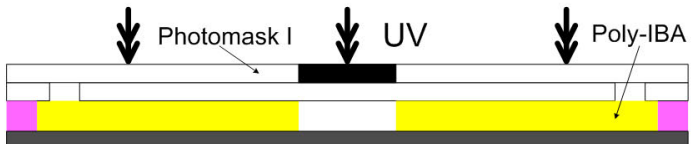

(c)

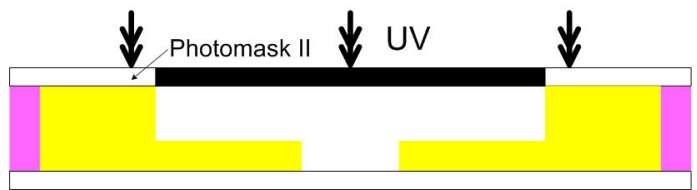

(d)

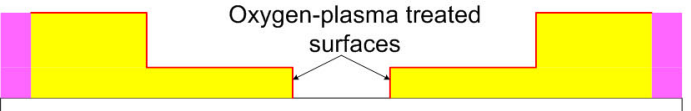

(e)

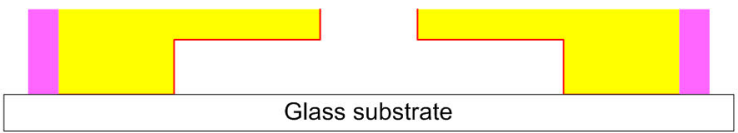

(f)

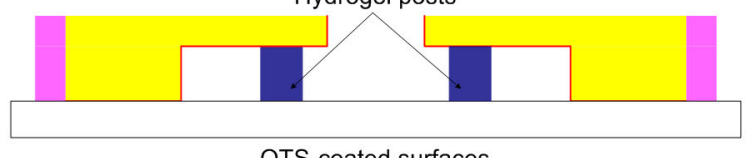

(g)

(h)

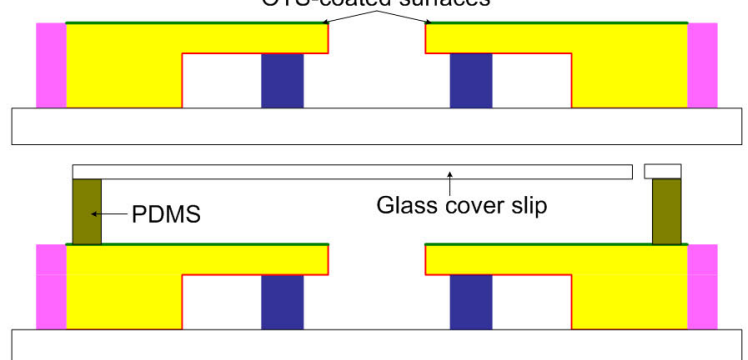

Figure 4: Fabrication process flow of an IR-light actuated tunable-focus microlens.

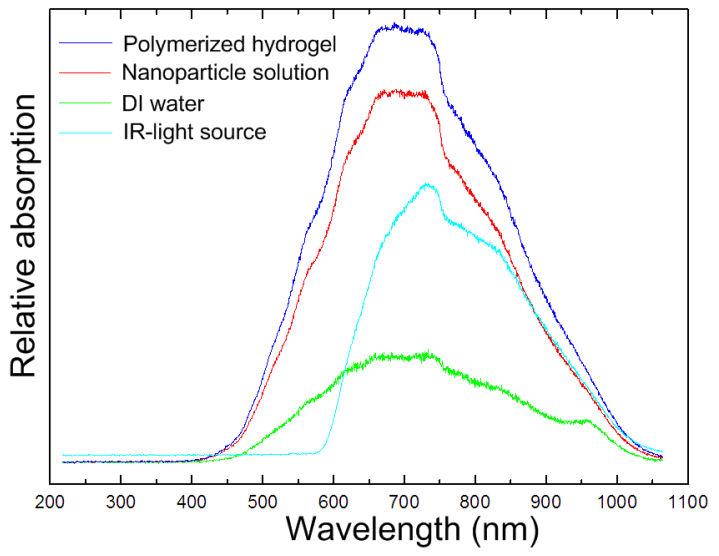

Figure 5: Absorption spectra of the gold nanoparticle solution, the polymerized IR-light-responsive hydrogel with entrapped gold nanoparticles, DI water and the IR-light source.

\section{Contact Angles of Surfaces}

Surfaces of poly-IBA are treated by oxygen plasma and OTS. The contact angles of DI water on the surfaces of natural, oxygen-plasma treated and OTS-coated poly-IBA are measured by a goniometer and are $105^{\circ}, 47^{\circ}$ and $116^{\circ}$, respectively. With
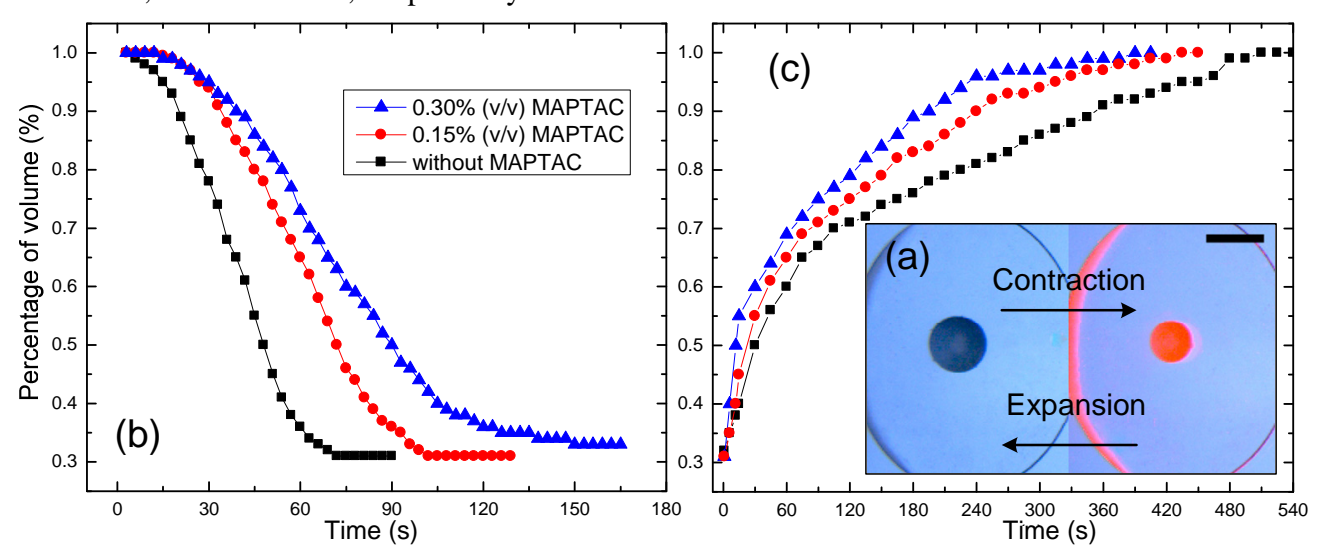

Figure 6: (a) IR-light-responsive hydrogel changes from the expanded state (left) to the contracted state (right) under IR-light irradiation. (b) Contraction and (c) expansion time of IR-light-responsive hydrogel with three different concentrations of MAPTAC. oxygen-plasma treatment, the surface is rendered hydrophilic; with OTS coating, the surface is more hydrophobic than untreated.

\section{Response Times of Hydrogel}

IR-light-responsive hydrogel begins to contract when the temperature increases from lower than to above the lower critical solution temperature (LCST) of hydrogel [10]. A small amount of an ionizable monomer, 3-(methacryloylamino) propyl trimethylammonium chloride (MAPTAC) is added into IR-light-responsive hydrogel pre-polymer solution to increase the LCST of hydrogel. Two concentrations of MAPTAC are mixed into the hydrogel pre-polymer solution and the LCST shifts from $32^{\circ} \mathrm{C}$ to $42^{\circ} \mathrm{C}(0.15 \% \mathrm{v} / \mathrm{v})$ and $48^{\circ} \mathrm{C}(0.30 \% \mathrm{v} / \mathrm{v})$, respectively.

Fig. 6 shows the contraction and expansion time of a hydrogel posts with different concentration of MAPTAC under IR-light irradiation. The IR-light-responsive hydrogel post is polymerized in a $250-\mu \mathrm{m}$ thick cavity under UV radiance (intensity, $I_{U V}=12.5$ $\mathrm{mW} / \mathrm{cm}^{2}$; time, $t=11 \mathrm{~s}$ ). The power intensity of the IR-light source at the surface of hydrogel posts is measured by an IR sensor and to be $1.42 \mathrm{~W} / \mathrm{cm}^{2}$. The full contraction of IR-light-responsive hydrogel post takes about ten seconds [Fig. 6 (b)], while the full expansion takes longer time due to the reliance on heat dissipation to cool down the hydrogel [Fig. 6 (c)]. 


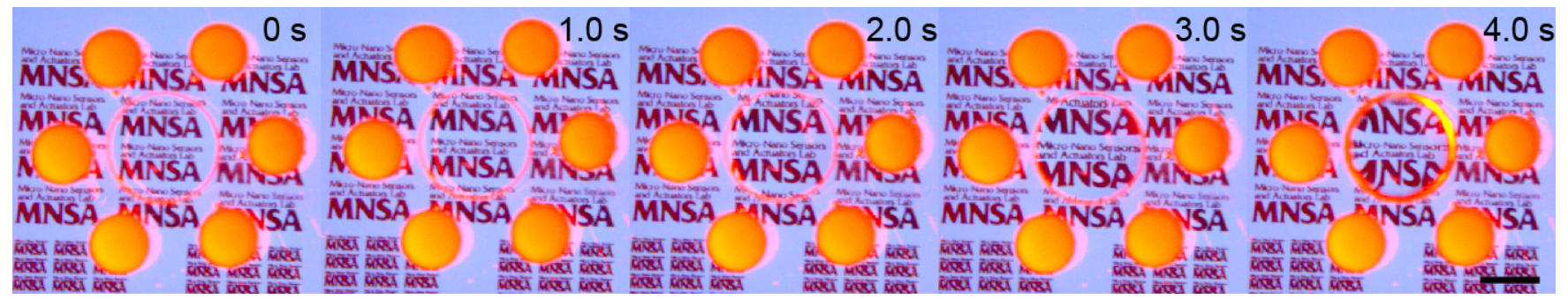

Figure 7: Sequence of frames taken from a video demonstrates the dynamic magnification using a tunable-focus microlens. The scale bar represents $1 \mathrm{~mm}$.

\section{Optical Properties of Tunable-Focus Microlenses}

To reduce the response time of the microlens, multiple hydrogel posts are used and the volume change in each post adds up. Here, the focal length of the microlens changes from divergent to convergent in 4 seconds under IR-light irradiation.

Fig. 7 shows the image magnification using a microlens with six hydrogel posts by tuning its focal length. The observed image plate with MNSA logo, printed on a transparency film, is located 1.0 mm under the back side of the device substrate. A CCD-coupled stereoscope is placed above the microlens to record the video. The IR-light lamp is irradiated from an oblique angle and the power intensity at the plate of IR-responsive hydrogel posts is measured to be $1.42 \mathrm{~W} / \mathrm{cm}^{2}$.

Initially, the microlens is slightly divergent and the image is slightly reduced. At the time instant of $1.0 \mathrm{~s}$, the water-oil interface becomes flat and the image is the same size as the object. From 1.0 $\mathrm{s}$ on, the microlens becomes convergent; thus its focal length decreases and the resultant image is gradually magnified. For the convergent microlens, the focal length is measured by determining optically the minimum focused point of a collimated input light beam along the optical axis. At $4.0 \mathrm{~s}$, the microlens reaches its minimum focal length $(f=8.8 \mathrm{~mm}$, limited by the height of the current water container). A close-to-flat starting profile of the microlens [Fig. 1(b)] can further decrease the tuning time needed from divergent to convergent.

The spherical aberration of the microlens calculated from the profile shown in Fig. 2(b) using an optical simulation tool (Zemax) is $-0.245 \mu \mathrm{m}$.

\section{CONCLUSION}

A tunable-focus microlens driven by IR-light in seconds for fiber endoscopy is presented. A liquid microlens is formed through the water-oil interface and pinned at the $\mathrm{H}-\mathrm{H}$ boundary at the top edge of the aperture. Under IR-light irradiation, the total water volume in the water container is changed by IR-light-responsive hydrogel, varying the focal length of the microlens from $-17.4 \mathrm{~mm}$ to $8.8 \mathrm{~mm}$ in 4 seconds.

In future studies, the hydrogel posts can be directly patterned and actuated by, thus self-aligned to, optical fibers; hence the demonstrated microlenses can be intrinsically integrated with fiber endoscopes. To reduce the response time of the microlens to sub-seconds, more hydrogel posts can be fabricated and different hydrogels can be used [11]. To enhance the mechanical robustness of the microlenses, more robust $\mathrm{H}-\mathrm{H}$ boundaries will be investigated. The microlenses can be extended to tunable microlens arrays for larger field of view. These microlenses may also be used in other optical imaging applications.

\section{ACKNOWLEDGEMENTS}

This work was supported by NSF (ECCS 0702095) and Wisconsin Institutes for Discovery. The authors thank Richard K. Noll for assistance in taking SEM images, Professor David Beebe for access to his facilities, Professor Franco Cerrina for access to Zemax, and Professor Leon McCaughan, Chad Staus, Chi-Wei Lo and Professor Liang Dong for their insightful discussions.

\section{REFERENCE}

1. B. I. Hirschowitz, L. E. Curtiss, C. W. Peters, and H. M. Pollard, "Demonstration of a new gastroscope, the fiberscope", Gastroenterology, 35, 50, (1958).

2. H. Jiang and L. Dong, "Smart lenses", Phys. World, 19, 29, (2006).

3. D. J. Beebe, J. S. Moore, J. M. Bauer, Q. Yu, R. H. Liu, C. Devadoss, and B. H. Jo, "Functional hydrogel structures for autonomous flow control inside microfluidic channels", Nature, 404, 588, (2000).

4. L. Dong, A. K. Agarwal, D. J. Beebe, and H. Jiang, "Adaptive liquid microlenses activated by stimuli-responsive hydrogels", Nature, 442, 551, (2006).

5. S. R. Sershen, G. A. Mensing, M. Ng, N. J. Halas, D. J. Beebe, and J. L. West, "Independent optical control of microfluidic valves formed from optomechanically responsive nanocomposite hydrogels", Adv. Mater., 17, 1366, (2005).

6. M. Brust, M. Walker, D. Bethell, D. J. Schiffrin, and R. Whyman, "Synthesis of Thiol-Derivatized Gold Nanoparticles in a 2-Phase Liquid-Liquid System", J. Chem. Soc., Chem. Commun., 801, (1994).

7. A. H. Latham and M. E. Williams, "Versatile routes toward functional, water-soluble nanoparticles via trifluoroethylester-PEG-thiol ligands", Langmuir, 22, 4319, (2006).

8. A. K. Agarwal, S. S. Sridharamurthy, D. J. Beebe, and H. R. Jiang, "Programmable autonomous micromixers and micropumps", J. Microelectromech. Syst., 14, 1409, (2005).

9. D. J. Beebe, J. S. Moore, Q. Yu, R. H. Liu, M. L. Kraft, B. H. Jo, and C. Devadoss, "Microfluidic tectonics: A comprehensive construction platform for microfluidic systems", Proc. Nat. Acad. Sci. U.S.A., 97, 13488, (2000).

10. J. Wang, Z. Y. Chen, M. Mauk, K. S. Hong, M. Y. Li, S. Yang, and H. H. Bau, "Self-actuated, thermo-responsive hydrogel valves for lab on a chip", Biomed Microdevices, 7, 313, (2005).

11. A. Richter, D. Kuckling, S. Howitz, T. Gehring, and K. F. Arndt, "Electronically controllable microvalves based on smart hydrogels: Magnitudes and potential applications", J. Microelectromech. Syst., 12, 748, (2003). 